\title{
On the solvatochromism, dimerization and tautomerism of indazole
}

\author{
Javier Catalán \\ Departamento de Química Física Aplicada, Universidad Autónoma de Madrid, \\ Cantoblanco, E-28024 Madrid, Spain \\ E-mail: javier.catalan@uam.es
}

Dedicated to Prof. Rosa Maria Claramunt on the occasion of her 65th anniversary

\begin{abstract}
A careful study of the solvatochromism of indazole was for the first time conducted in this work. The study revealed that indazole solvatochromism is governed mainly by the polarizability of the medium and, to a lesser extent, by its acidity and basicity. Based on the results of the solvatochromic analysis and their processing with Abe's model, this polar compound undergoes no significant change in dipole moment from its ground electronic state to its first excited state, which contradicts the predictions of other authors. Indazole dimerizes in both 2-methylbutane and 1-chlorobutane. However, neither its monomer nor its dimer undergoes tautomerization to $2 \mathrm{H}$-indazole in the ground state in response to an increase in dipolarity of the medium. Also, based on experimental evidence, no tautomerization occurs in the first excited singlet or triplet of the compound.
\end{abstract}

Keywords: Indazole, solvatochromism, dimerization, tautomerization.

\section{Introduction}

Indazole is a polar asymmetric bicyclic diazole heterocycle with a dipole moment in the gas phase of 1.76 D. ${ }^{1}$ It contains a basic pyridine nitrogen with an intrinsic basicity in the gas phase of $214.2 \mathrm{kcal} / \mathrm{mol}$ and an acidic pyrrole nitrogen with an intrinsic basicity in protonated form of $348.4 \mathrm{kcal} / \mathrm{mol}^{2}$. The fact that these two nitrogen sites are located in adjacent positions in the molecular structure (see Scheme 1) allows indazole at low concentrations in mildly acid or basic media to form $1 H$ and $2 H$ tautomers via acid-base interactions (see Scheme 1 ), ${ }^{3-10}$ as well as symmetrical dimers by hydrogen bonding. ${ }^{11,12}$ 


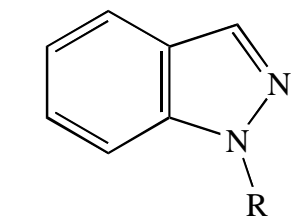

$1 H$-tautomer $(\mathrm{R}=\mathrm{H})$

1-methylindazole $(\mathrm{R}=\mathrm{Me})$

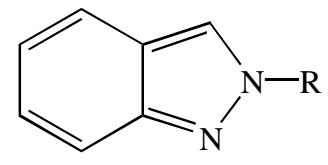

$2 H$-tautomer $(\mathrm{R}=\mathrm{H})$

2-methylindazole $(\mathrm{R}=\mathrm{Me})$

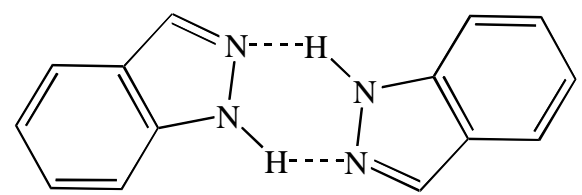

Indazole dimer

\section{Scheme 1}

Despite the wide range of potential interactions between dissolved indazole and solvents, few authors have shown interest in investigating its solvatochromism. In fact, a literature search for references to this topic retrieved just a single paper by Saha and Dogra entitled "Solvatochromic effects in the absorption and fluorescence spectra of indazole and its amino derivatives" where they examine the behaviour of the solute in only four solvents, namely: cyclohexane, acetonitrile, methanol and water. ${ }^{13}$ These authors concluded that the dipole moment of indazole increases from $1.62 \mathrm{D}$ in the ground electronic state to $2.44-2.96 \mathrm{D}$ in its first excited singlet (i.e. that the dipole moment is nearly doubled upon excitation to the first excited singlet).

Although $1 \mathrm{H} \leftrightarrow 2 \mathrm{H}$ tautomerism of indazole in the ground state was previously ruled out, ${ }^{9-12}$ according to Hirota et al. it occurs in the first triplet state of the indazole - benzoic acid complex, ${ }^{14}$ and in the first excited singlet of the indazole - acetic acid complex. ${ }^{15}$ They ascribed both instances of tautomerism to a double proton transfer in the complexes. In the present author's opinion, however, there is the scarcely explored possibility that the solvent dipolarity might enable indazole tautomerism since 1-methylindazole possesses a dipole moment of 1.5 $\mathrm{D},{ }^{16}$ whereas 2-methylindazole has one of $3.4 \mathrm{D},{ }^{16}$ and is therefore considerably more polar.

The dimerization of indazole by double hydrogen bonding produces a symmetric dimer which, according to Hirota et al., ${ }^{14,15}$ might facilitate the formation of the $2 \mathrm{H}$ tautomer -at least via its excited electronic state -and that of the dimer tautomer in highly polar media.

In this work, we studied the solvatochromism of indazole in 22 different solvents of variable dipolarity (SdP), polarizability (SP), acidity (SA) and basicity (SB) as measured on the empirical scales previously developed by our research group ${ }^{17-20}$ and compared the results with those for 1methylindazole. Measurements were additionally made in 1-chlorobutane $(\mathrm{ClB})$ at temperatures from 273 to $153 \mathrm{~K}$ and in 2-methylbutane (2MB) at 298-248 $\mathrm{K}$ in order to assess the sensitivity of indazole to the polarizability and dipolarity of the medium. We also examined the dimerization of indazole and its potential tautomerization. Finally, we used Abe's model ${ }^{21}$ to quantify the change in dipole moment upon electronic excitation from $S_{0}$ to $S_{1}$. 


\section{Results and Discussion}

We first examined the results of the solvatochromic analysis of indazole in 21 different solvents and the gas phase, and those of its thermochromic analysis in $\mathrm{ClB}$ at $283-153 \mathrm{~K}$ and $2 \mathrm{MB}$ at 298-248 K, and then compared them with those of the solvatochromic analysis of 1methylindazole in 11 different solvents and the gas phase. Finally, we used Abe's method ${ }^{21}$ to assess polarizability and dipole moment changes in indazole from the ground state $\left(S_{0}\right)$ to the first excited state $\left(S_{1}\right)$, and examined the potential dimerization and tautomerization of the compound in dilute solutions in $\mathrm{ClB}$ and $2 \mathrm{MB}$.

\section{On the solvatochromism of indazole}

Table 1. Wave numbers (in $\mathrm{cm}^{-1}$ ) of the $0-0$ component of the first absorption band of indazole and of 1-methylindazole in the studied solvents ${ }^{a}$

\section{Indazole}

\begin{tabular}{cccc}
\hline Solvent & $\mathbf{v}\left(\mathrm{cm}^{-1}\right)$ & Solvent $^{b}$ & $\mathbf{v}\left(\mathrm{cm}^{-1}\right)$ \\
\hline gas-phase & 34407 & 2,2,2-trifluoroethanol & 33918 \\
Perfluorohexane & 34266 & acetic acid & 33711 \\
$n$-pentane & 33986 & 2-methylbutane (2MB) 293 & 34005 \\
$n$-hexane & 33973 & $2 \mathrm{MB} 283$ & 34000 \\
Decalin & 33880 & $2 \mathrm{MB} 273$ & 33995 \\
diethyl ether & 33776 & $2 \mathrm{MB} 263$ & 33989 \\
di- $n$-butyl ether & 33727 & $2 \mathrm{MB} 253$ & 33981 \\
1,4-dioxane & 33732 & $2 \mathrm{MB} 243$ & 33968 \\
Tetrahydrofuran & 33715 & 1-chlorobutane $(\mathrm{ClB}) 293$ & 33881 \\
ethyl acetate & 33818 & ClB273 & 33875 \\
Dichloromethane & 33808 & ClB253 & 33867 \\
Chloroform & 33735 & ClB233 & 33856 \\
Methanol & 33732 & ClB213 & 33848 \\
Ethanol & 33701 & ClB193 & 33840 \\
1-propanol & 33686 & ClB173 & 33827 \\
1-butanol & 33650 & ClB153 & 33808 \\
Water & 33792 & acetonitrile & 33864 \\
\hline
\end{tabular}

${ }^{a}$ The derivative function was used while estimating the maximum absorption wavelength for the 0-0 component. ${ }^{b}$ Temperature $(\mathrm{K})$ is indicated; room temperature $(293 \mathrm{~K})$ can be assumed, otherwise. 
1-Methylindazole

\begin{tabular}{cccc}
\hline Solvent & $\mathbf{v}\left(\mathrm{cm}^{-1}\right)$ & Solvent & $\mathbf{v}\left(\mathrm{cm}^{-1}\right)$ \\
\hline gas-phase & 33369 & di- $n$-butyl ether & 32848 \\
Perfluorohexane & 33202 & methanol & 32885 \\
$n$-pentane & 32920 & ethanol & 32870 \\
$n$-hexane & 32897 & 1-propanol & 32829 \\
Decalin & 32799 & 1-butanol & 32844 \\
diethyl ether & 32891 & acetic acid & 32828 \\
\hline
\end{tabular}

Table 1 shows the energy, in $\mathrm{cm}^{-1}$, of the $0-0$ component of the first absorption band of indazole in the different solvents as the starting point for its solvatochromic study. The results provided by the solvatochromic scales conformed to the following equation (see Figure 1):

$$
v=-(786 \pm 39) \mathrm{SP}-(128 \pm 20) \mathrm{SA}-(321 \pm 23) \mathrm{SB}+(34470 \pm 25)
$$

with $n=34, r=0.982$ and $\mathrm{sd}=31 \mathrm{~cm}^{-1}$.

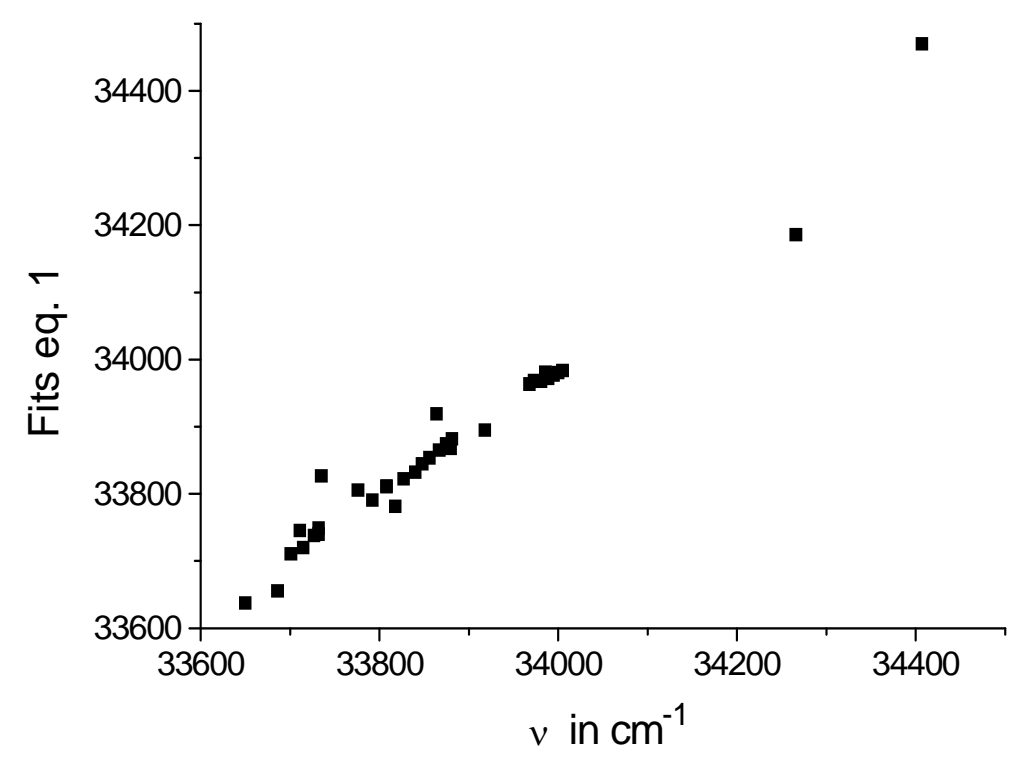

Figure1. Fits from Equation $1(=-786 \mathrm{SP}-128 \mathrm{SA}-321 \mathrm{SB}+34470) v s$ the corresponding experimental $v$ data values from Table 1.

Rather unexpectedly, the solvatochromism of such a polar molecule as indazole is insensitive to the dipolarity SdP of the medium. As a result, its electronic states $S_{0}$ and $S_{1}$ should possess an identical dipole moment, which contradicts previous findings of Saha and Dogra. ${ }^{13}$ We shall return to this point later. Also, the independent term of the equation, $34470 \pm 25 \mathrm{~cm}^{-1}$, is quite 
consistent with the value $34471.691 \pm 0.006 \mathrm{~cm}^{-1}$ obtained by Berden et al. ${ }^{22}$ using rotationally resolved ultraviolet spectroscopy in a supersonic jet.

Based on Equation 1, the solvatochromism of indazole depends mainly on the polarizability SP of the medium and also, to a lesser extent, on its basicity SB, and even less so on its acidity SA. These specific contributions to the solvatochromism of indazole expose the roles of its pyrrole nitrogen (an acid site) and pyridine nitrogen (a basic site).

One interesting way of confirming the solvatochromic behaviour of indazole would be by suppressing its acid site (i.e. by using its 1-methyl derivative [Scheme 1] instead of the parent compound). The energies of the $0-0$ component of the first absorption band for 1-methylindazole in 11 different solvents and in the gas phase are included in Table 1. The results obtained from the solvatochromic scales ${ }^{17}$ fitted the following equation:

$$
v=-(811 \pm 45) \mathrm{SP}-(61 \pm 34) \mathrm{SA}+(33404 \pm 26)
$$

with $n=12, r=0.988$ and $\mathrm{sd}=29 \mathrm{~cm}^{-1}$.

As can be seen from Equation 2, the solvatochromism of 1-methylindazole is virtually exclusively dependent on the polarizability (SP) of the medium. Also, it is scarcely dependent on its acidity (SA); in fact, the SA coefficient decreased markedly from indazole to 1-methylindazole $(-128 \pm 20 v s-61 \pm 34)$, which suggests that the presence of a methyl group in the derivative may hinder interaction between acid solvents and the neighbouring basic nitrogen site. The assumption that the solvatochromism of 1-methylindazole is virtually exclusively governed by the solvent polarizability (SP) is supported by the small statistical deterioration observed in the fitting of Equation 3, which, unlike Equation 2, excludes the influence of acidity (SA) on 1-methylindazole:

$$
v=-(835 \pm 47) \mathrm{SP}+(33405 \pm 28)
$$

with $n=12, r=0.984$ and $\mathrm{sd}=32 \mathrm{~cm}^{-1}$.

\section{Dipole moment of indazole in its $1\left(\pi, \pi^{*}\right)^{1}$ state}

Based on the above-discussed solvatochromic analysis, we deduce that the dipole moments of indazole and its 1-methyl derivative do not change from the ground electronic state to the first excited state. In order to confirm this crucial hypothesis, we used Abe's approach as formulated via the following equation (Equation 4) to assess changes in dipole moment and isotropic polarizability in indazole on electronic photoexcitation:

$$
\left[\left(\mu_{i}\right)^{2}-\left(\mu_{0}\right)^{2}\right]+\left(\alpha_{i}\right) \cdot A=B
$$

These parameters, which were calculated from the intercept and slope, respectively, of a $B v s A$ plot. $\mu_{i}$ and $\mu_{0}$ in Equation 4, represent the dipole moment of the chromophore in the excited and ground state, respectively, which are involved in the electronic transition, and $\alpha_{i}$ is the isotropic polarizability of the excited state. $A$ and $B$ were evaluated from the molecular mass, density, refraction index and dielectric constant for the solvents, as well as from the ionization potential 
and electronic transition energy for indazole as formulated in Abe's expressions, which are given in the Experimental Section.

Applying Abe's method to the energy of the $0-0$ component of the first absorption band for indazole in the different solvents provided an excellent fit $(r=0.9999$, Figure 2$)$, with a slope of $(274.4 \pm 0.3) \times 10^{-25} \mathrm{~cm}^{3}-$ the isotropic polarizability for the $1\left(\pi, \pi^{*}\right)^{1}$ state - and an intercept of $(-0.11 \pm 0.13) \times 10^{-36} \mathrm{erg} \mathrm{cm}^{3}$ - the corresponding dipole moment is $\mu\left[1\left(\pi, \pi^{*}\right)^{1}\right]=1.73 \pm 0.13$ D. The dipole moment was calculated from the intercept, which was set equal to $\left(\mu^{*}\right)^{2}-\left(\mu_{0}\right)^{2}$ in order to obtain $\mu_{0}=1.76 \pm 0.02 \mathrm{D}^{1}$ for indazole. A total of 14 solvents were used (the $\mathrm{ClB}$ at 293-153 K and $2 \mathrm{MB}$ at 293-243 K). Therefore, within experimental error, the two dipole moments can be considered identical, which confirms the previous assumption from the solvatochromic results, i.e. that the solvatochromism of indazole is independent of the dipolarity of the medium (see Equation 1).

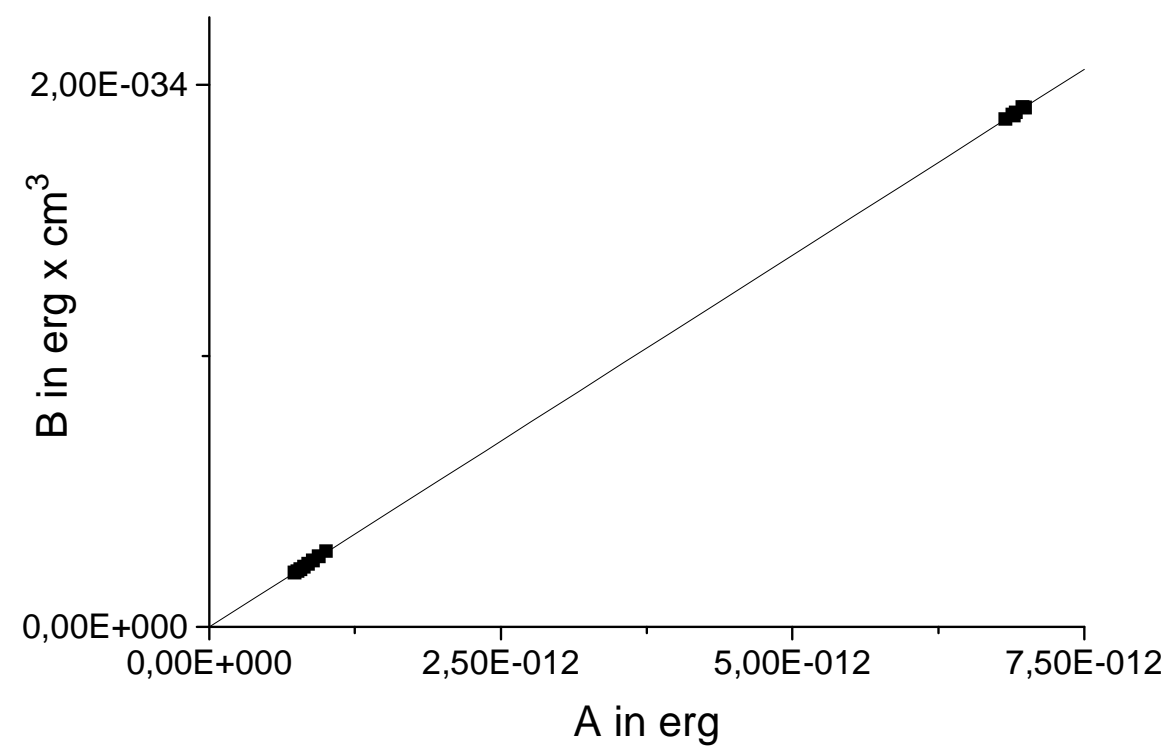

Figure 2. Plot of $B$ against $A$ following Abe's model for indazole.

The unexpected strong dependence of the $S_{0} \rightarrow S_{1}$ transition of indazole on the solvent polarizability warrants seeking a straightforward explanation, which might be as follows: the transition promotes one of the electrons in the pair contributed by the pyrrole nitrogen to the $\pi$ cloud and causes its delocalization over the bicyclic system. As a result, the excited compound possesses a substantially increased polarizability that leads to a bathochromic shift in its first transition state by the effect of the polarizability of the medium. This hypothetical increase in polarizability is supported by the fact that, based on $\mathrm{mp} 2$ calculations with a $6-31 \mathrm{G}^{* *}$ basis set, indazole in its ground electronic state possesses an isotropic polarizability of $112.1 \times 10^{-25} \mathrm{~cm}^{3}$ 
${ }^{23}$ and, based on calculations using Abe's method, one of $274.4 \pm 0.3 \times 10^{-25} \mathrm{~cm}^{3}$ (i.e. 2.4 times higher) in its $1\left(\pi, \pi^{*}\right)^{1}$ state.

No doubt, the proposed mechanism warrants careful investigation, which at the moment is in progress.

\section{On the dimerization of indazole}

Because indazole is a polar substance $(\mu=1.76 \mathrm{D})$ containing an acid site (pyrrole nitrogen) and a basic site (pyridine nitrogen) at adjacent positions, it tends to form a non-polar symmetric doubly hydrogen bonded dimer at low concentrations in inert solvents.

Figure 3 illustrates the thermochromic behaviour of a $3.6 \times 10^{-5} \mathrm{M}$ solution of indazole in a highly inert solvent such as $2 \mathrm{MB}$. As can be seen in Figure 4, as the solution temperature was lowered, the chromophore exhibited an unexpected bathochromic shift of $c a .268 \mathrm{~cm}^{-1}$ in the region from 248 to $223 \mathrm{~K}$ clearly indicating a structural change in the compound. Jalviste et al. ${ }^{11}$ found the shift resulting from the dimerization of indazole at a low temperature in the gas phase to be $321 \mathrm{~cm}^{-1}$.

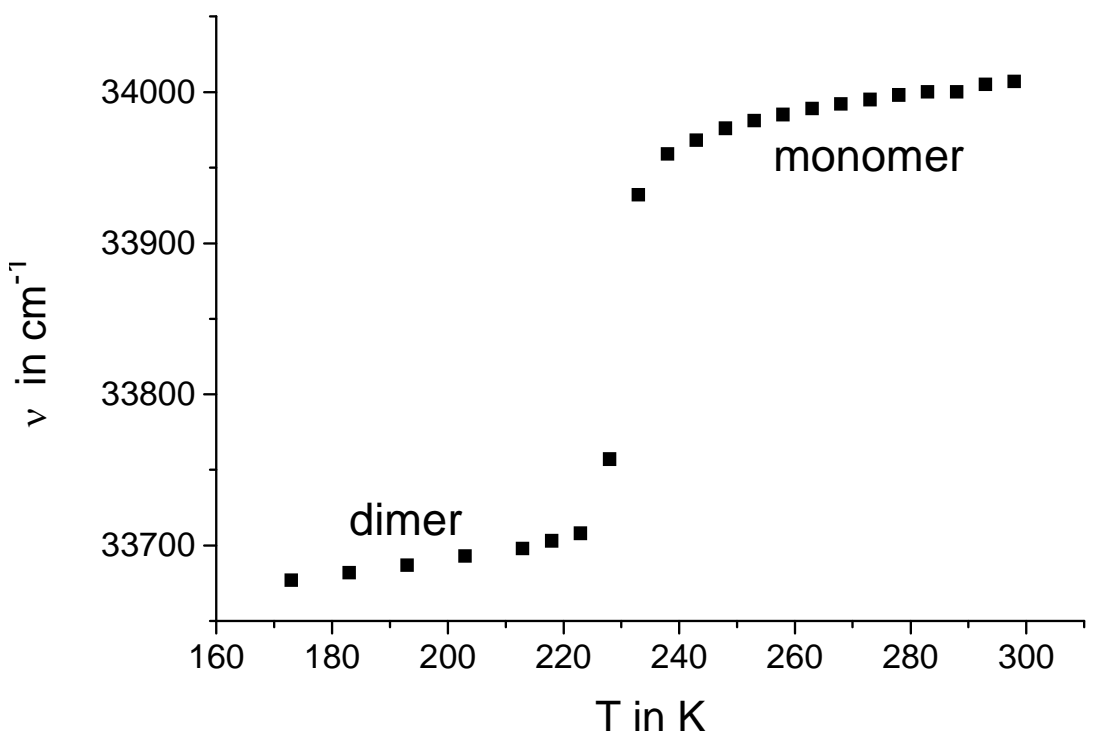

Figure 3. Thermochromic behaviour of a $3.6 \times 10^{-5} \mathrm{M}$ solution of indazole in $2 \mathrm{MB}$. 


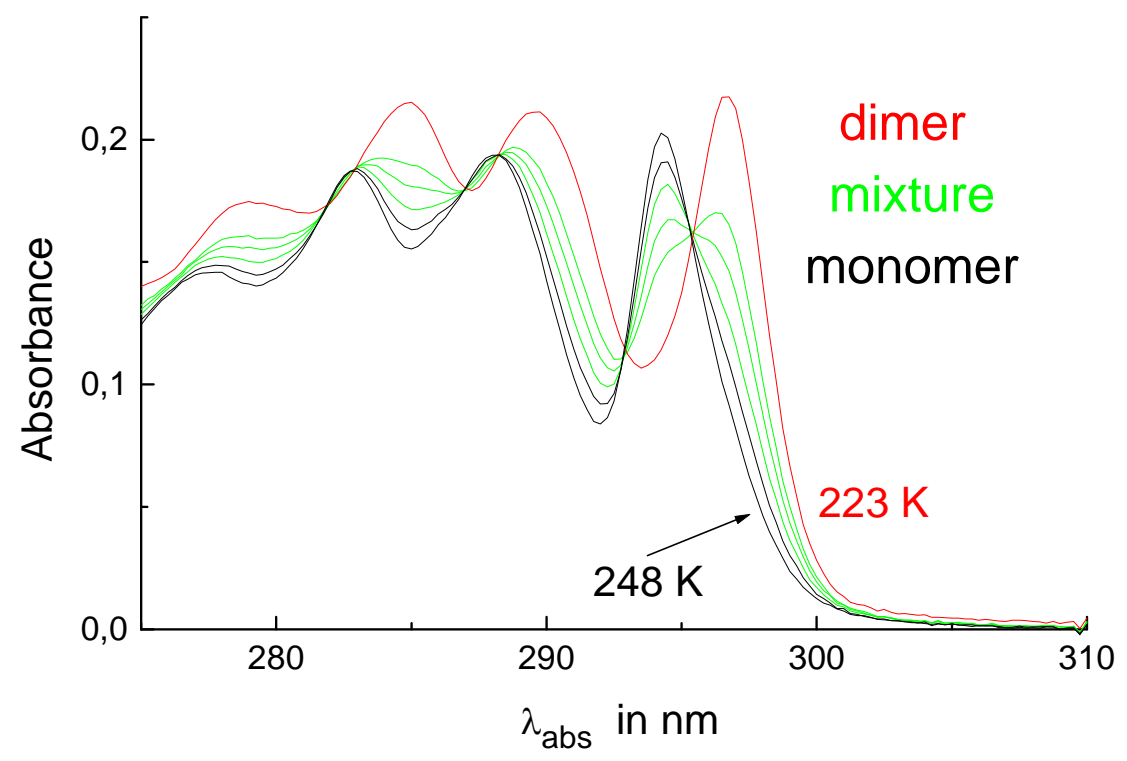

Figure 4. Absorption spectra of indazole in $2 \mathrm{MB}$ at 248, 243, 238, 233, 228 and $223 \mathrm{~K}$.

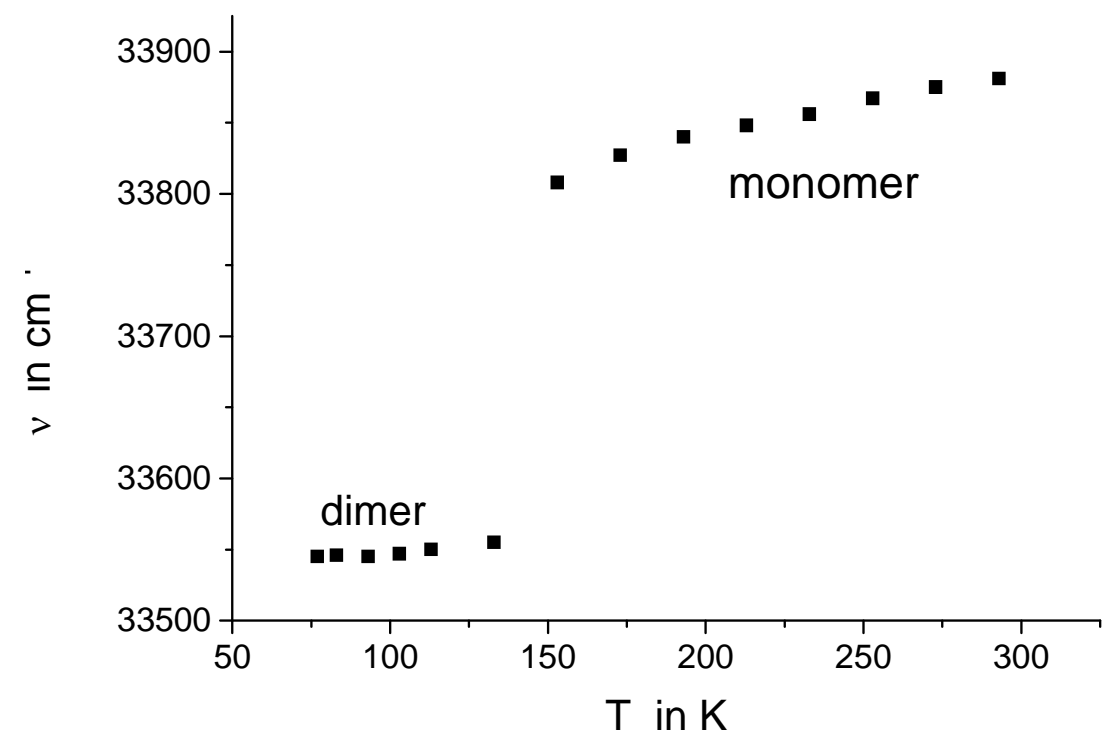

Figure 5. Thermochromic behaviour of a $3.6 \times 10^{-5} \mathrm{M}$ solution of indazole in ClB. 
Figure 5 illustrates the thermochromic behaviour of a $3.6 \times 10^{-5} \mathrm{M}$ solution of indazole in $\mathrm{ClB}$. The figure affords two interesting conclusions, namely:

No change in indazole is apparent from its thermochromic behaviour over the temperature range 293-153 K.

Below $153 \mathrm{~K}$, the compound exhibits a thermochromic change of $253 \mathrm{~cm}^{-1}$ indicating dimerization.

\section{On the tautomerization of indazole}

In recent work, we showed the dipolarity SdP of ClB to increase markedly from $293 \mathrm{~K}(0.523)$ to $77 \mathrm{~K}(1.294){ }^{24}$ Taking into account that our SdP dipolarity scale ${ }^{17}$ spans the range from 0 for the gas phase to 1 for such a highly polar solvent as DMSO, lowering the temperature of the ClB solution of indazole must obviously have exposed the compound to a strong dipolarity change. As noted in the Introduction, the tautomerization of indazole from its less polar form $(1 H$, $\mu_{1 \text { meindazole }}=1.5 \mathrm{D}^{16}$ to its more polar form $\left(2 \mathrm{H}, \mu_{2 \text { meindazole }}=3.4 \mathrm{D}^{16}\right)$ can be facilitated by increasing the polarity of the solvent. As can be seen from Figure 5, neither the segment corresponding to the presence of the monomer (293-153 K) nor that corresponding to the doubly hydrogen bonded dimer suggest that raising the polarity of the medium leads to tautomerization from the $1 H$ form to the $2 H$ form in the ground electronic state.

In 1983, Hirota et al. ${ }^{14}$ reported that the first excited triplet of the indazole:benzoic acid complex comprises both tautomers $(1 H$ and $2 H)$. Also, in 1985 they reported that the first excited singlet of the indazole:acetic acid complex also exhibits tautomerization, via double hydrogen bonding, and hypothesized that such a singlet was very probably the origin of the tautomerism subsequently detected in the triplet state. ${ }^{15}$

Figure 6 shows the emission spectra for the previous $\mathrm{ClB}$ solution of indazole over the temperature range $293-113 \mathrm{~K}$. The figure affords some interesting conclusions about the thermochromic behaviour of the compound, namely:

(a) The fluorescence spectra obtained at temperatures from 293 to $173 \mathrm{~K}$ exhibit no sign of a thermochromic change in indazole.

(b) Below $173 \mathrm{~K}$, the solution exhibits a thermochromic change indicating dimerization of the compound.

(c) Interestingly, indazole dimerization occurs at a temperature about $20 \mathrm{~K}$ higher in the emission spectrum than in the absorption spectrum (i.e. the process is more favourable in the excited singlet state than in the ground state).

(d) The spectra do not preclude a potential tautomerization of indazole monomer or dimer as the dipolarity SdP of the medium is increased. 


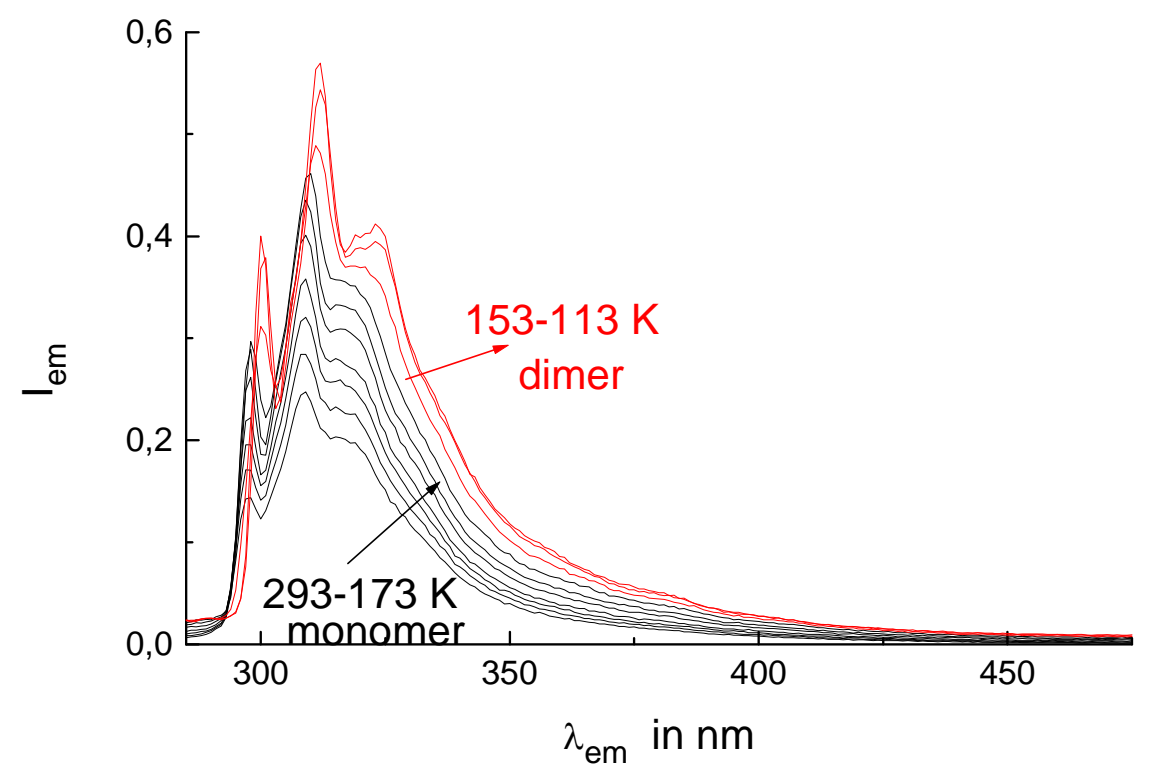

Figure 6. Emission spectra for $3.6 \times 10^{-5} \mathrm{M}$ solution of indazole in $\mathrm{ClB}$ at 293-113 K. $\lambda_{\mathrm{exc}}=277$ nm.

Figure 7 shows the emission spectrum for the previous solution at $93 \mathrm{~K}$. The spectrum clearly exhibits the phosphorescence of indazole dimer but its fluorescence has the same envelope as at 113 K (see Figure 6).

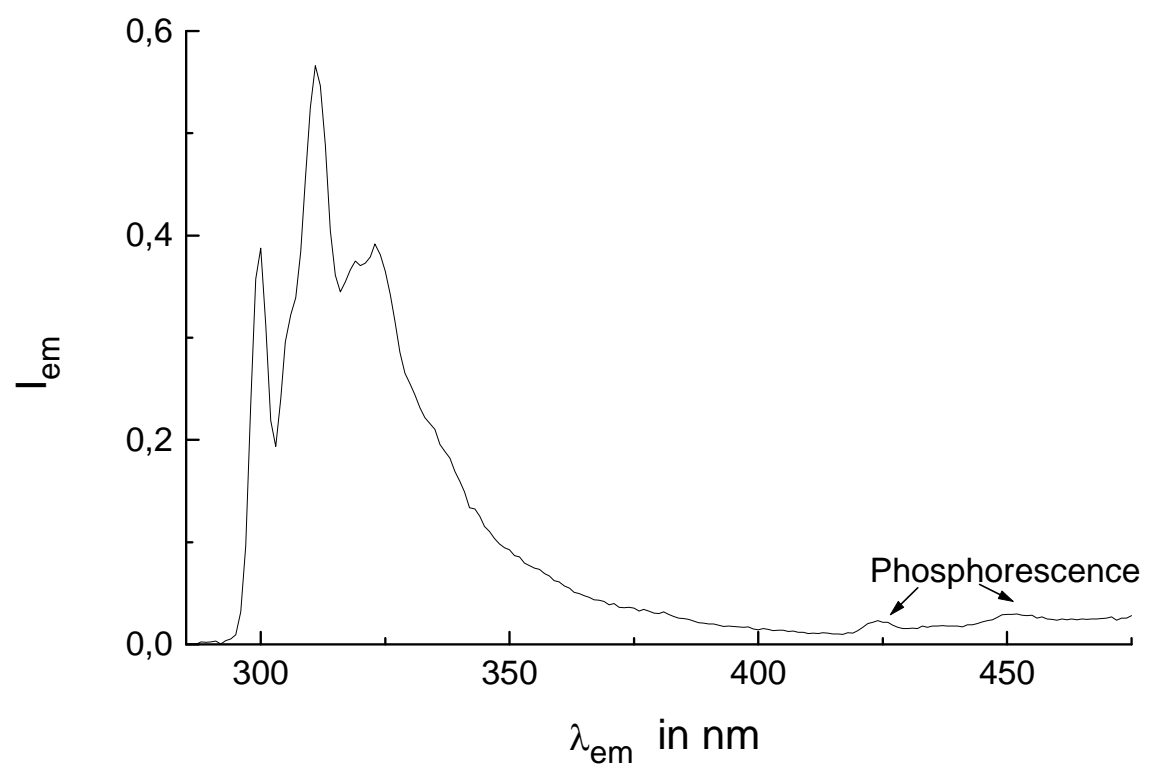

Figure 7.- Emission spectra of a $3.6 \times 10^{-5} \mathrm{M}$ solution of indazole in $\mathrm{ClB}$ at $93 \mathrm{~K} . \lambda_{\text {exc }}=277$ nm. 
Figure 8 shows the phosphorescence spectra for indazole, 1-methylindazole and 2methylindazole in $\mathrm{ClB}$ at $77 \mathrm{~K}$ as obtained by using an excitation wavelength of $290 \mathrm{~nm}$ and a detection delay of $280 \mu \mathrm{s}$. Clearly, the phosphorescence of indazole contains no emission band due to the $2 H$ tautomer.

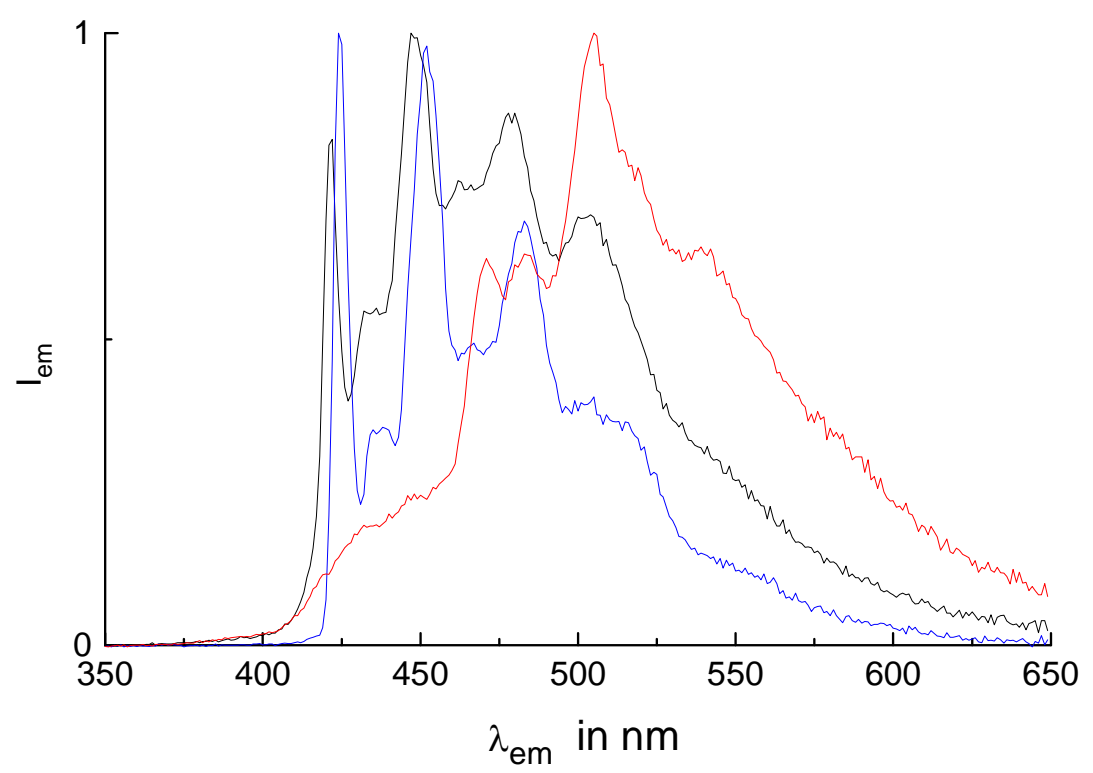

Figure 8. Normalized Phosphorescence spectra of indazole (black), 1-methylindazole (blue), and 2-methylindazole (red).

\section{Conclusions}

Examining the solvatochromism of the $S_{0} \rightarrow S_{1}$ transition in indazole allowed us to identify the singular effect of the delocalization of the $\pi$ electron pair contributed by the pyrrole nitrogen, which considerably increases the polarizability of its $1\left(\pi, \pi^{*}\right)^{1}$ state.

That indazole undergoes dimerization is quite apparent from its thermochromism in a dilute solution in a polar $(\mathrm{ClB})$ or non-polar solvent $(2 \mathrm{MB})$. By contrast, no signs of tautomerization in the ground, $1\left(\pi, \pi^{*}\right)^{1}$ or $1\left(\pi, \pi^{*}\right)^{3}$ states exist for this compound in its monomeric or dimeric forms.

\section{Experimental Section}

General. The 1-chlorobutane (ClB) used was Chromasolv-grade (purity $99.8 \%$; b.p. $77-78{ }^{\circ} \mathrm{C}$ ) and contained $0.001 \%$ or less moisture; 2-methylbutane (2MB) was Merck Uvasol-grade (purity $\geq 99.5 \%$; b.p. $30{ }^{\circ} \mathrm{C}$ ), 1,4-dioxane, 1-propanol and 1-butanol were Aldrich spectrophotometricgrade (purity 99\%+); perfluorohexane, decalin, and 2,2,2-trifluoroethanol were from Aldrich 
(purity 99\%); dibutyl ether was Aldrich reagent-grade (purity $+99 \%$ ); acetic acid was Sigma ACS Reagent Plus grade (purity 99.7\%); and acetonitrile, tetrahydrofuran, ethyl acetate, dichloromethane, chloroform, tetrachloromethane, $n$-hexane, $n$-heptane, methanol, ethanol and diethyl ether were Merck Uvasol-grade (purity $\geq 99 \%$;). Bi-distilled water was used throughout. The preparations of indazole, 1-methylindazole, and 2-methyl-indazole are previously described. ${ }^{9}$

Temperatures in the range 77-293 K were controlled with an Oxford DN1704 cryostat equipped with an ITC4 controller interfaced to the spectrometers. Solutions were purged with dried nitrogen $99.99 \%$ pure.

All UV/Vis absorption spectra were recorded on a Cary-5 spectrophotometer at variable temperatures from 77 to $293 \mathrm{~K}$, using Suprasil quartz cells of $1 \mathrm{~cm}$ path length that were fixed to the cryostat.

Corrected fluorescence spectra were obtained with a calibrated Aminco-Bowman AB2 spectrofluorimeter. The sensitivity factors for the emission channel, which included not only those depending on the detector, but also those related to the emission monochromator and optical arrangement (channel emission included), were obtained by using the FP-123 correction kit from SLM Instruments. This required mounting a standard lamp in a channel at right angles to the emission channel in an OL $254 \mathrm{M}$ spectra irradiance lamp from Optronic Laboratories. The lamp was operated at a constant voltage supplied by an SP-270 power source the light output of which was driven into an integrating sphere with a pinhole leading to the fluorimeter emission channel. The conversion factors thus obtained allowed the measured spectra to be transformed into absolute spectra, which are instrument-independent.

In this work it is not used neither metal ion niether filter paper for facilitating inter system crossing to enhance phosphorescence. Alone we have used to detect the phosphorescence a detection delay of $280 \mu \mathrm{s}$.

The terms $\mathbf{A}$ and $\mathbf{B}$ of Abe's equation comes given by the expressions:

$\mathbf{A}=3 \times\left[\left(2\left(\varepsilon^{\mathrm{s}}-\left(\mathrm{n}^{\mathrm{s}}\right)^{2}\right)\left(\varepsilon^{\mathrm{s}}+\left(\mathrm{n}^{\mathrm{s}}\right)^{2}\right)\right) / \varepsilon^{\mathrm{s}}\left(\left(\mathrm{n}^{\mathrm{s}}\right)^{2}+2\right)^{2}+\left(\left(\mathrm{n}^{\mathrm{s}}\right)^{2}-1\right) /\left(\left(\mathrm{n}^{\mathrm{s}}\right)^{2}+2\right)\right]^{-1} \mathrm{x}[\mathrm{kt} \mathrm{x}$

$\left.\left.\left(\left(\varepsilon^{\mathrm{s}}-\left(\mathrm{n}^{\mathrm{s}}\right)^{2}\right)\left(2 \varepsilon^{\mathrm{s}}+\left(\mathrm{n}^{\mathrm{s}}\right)^{2}\right) / \varepsilon^{\mathrm{s}}\left(\left(\mathrm{n}^{\mathrm{s}}\right)^{2}+2\right)^{2}+0.5\left(\left(\mathrm{n}^{\mathrm{s}}\right)^{2}-1\right) /\left(\left(\mathrm{n}^{\mathrm{s}}\right)^{2}+2\right)\right)\right)\left(\mathrm{I}^{\mathrm{s}}(\mathrm{I}-\mathrm{hcv})\right) /\left(\mathrm{I}^{\mathrm{s}}+\mathrm{I}-\mathrm{hcv}\right)\right]$

and

$\mathbf{B}=3 \times\left[\left(2\left(\varepsilon^{\mathrm{s}}-\left(\mathrm{n}^{\mathrm{s}}\right)^{2}\right)\left(\varepsilon^{\mathrm{s}}+\left(\mathrm{n}^{\mathrm{s}}\right)^{2}\right)\right) / \varepsilon^{\mathrm{s}}\left(\left(\mathrm{n}^{\mathrm{s}}\right)^{2}+2\right)^{2}+\left(\left(\mathrm{n}^{\mathrm{s}}\right)^{2}-1\right) /\left(\left(\mathrm{n}^{\mathrm{s}}\right)^{2}+2\right)\right]^{-1} \mathrm{x}[\mathrm{kt} \mathrm{x}$

$\left.\left.\left(\left(\varepsilon^{\mathrm{s}}-\left(\mathrm{n}^{\mathrm{s}}\right)^{2}\right)\left(2 \varepsilon^{\mathrm{s}}+\left(\mathrm{n}^{\mathrm{s}}\right)^{2}\right) / \varepsilon^{\mathrm{s}}\left(\left(\mathrm{n}^{\mathrm{s}}\right)^{2}+2\right)^{2}+0.5\left(\left(\mathrm{n}^{\mathrm{s}}\right)^{2}-1\right) /\left(\left(\mathrm{n}^{\mathrm{s}}\right)^{2}+2\right)\right)\right) \times\left(\mathrm{I}^{\mathrm{s}} \times \mathrm{I} / \mathrm{I}^{\mathrm{s}}+\right)\right)\left(\alpha_{0}\right)-8.351$ $\mathrm{x} 10^{-42} \times\left(\rho^{\mathrm{s}} / \mathrm{M}^{\mathrm{s}}\right)^{1 / 3} \times \Delta v \times\left(\left((\mathrm{M} / \rho)^{1 / 3}+\left(\mathrm{M}^{\mathrm{s}} / \rho^{\mathrm{s}}\right)^{1 / 3}\right)^{-4}+\left((\mathrm{M} / \rho)^{1 / 3}+3\left(\mathrm{M}^{\mathrm{s}} / \rho^{\mathrm{s}}\right)^{1 / 3}\right)^{-4}+\left((\mathrm{M} / \rho)^{1 / 3}+5\right.\right.$ $\left.\left.\left.\left(\mathrm{M}^{\mathrm{s}} / \rho^{\mathrm{s}}\right)^{1 / 3}\right)^{-4}\right)^{-1}\right]$

where the notations s will refer to solvent.

The values of $\mathbf{A}$ and $\mathbf{B}$ can be calculated from observed values of : molecular weight (M), densities $(\rho)$, refraction index $(\mathrm{n})$, dielectric constant $(\varepsilon)$, ionization potential(I) and electronic transition energies $(v)$. 


\section{References}

1. Velino, B.; Cané, E.; Trombetti, A.; Corbelli, G.; Zerbetto, F.; Caminaty, W. J. Mol. Spectrosc. 1992, 155, 1-10.

http://dx.doi.org/10.1016/0022-2852(92)90543-W

2. Catalán, J.; Claramunt, R. M.; Elguero, J.; Laynez, J.; Menéndez, M.; Anvia, F.; Taagepera, M.; Taft, R. W. J. Am. Chem. Soc. 1988, 110, 4105-4111. http://dx.doi.org/10.1021/ja00221a001

3. Elguero, J.; Marzin, C.; Katrizky, A.R.; Linda, P., The Tautomerism of Heterocycles, Academic Press, New Jork, 1976.

4. Elguero, J. Pyrazoles and Their Benzo Derivatives, in Comprehensive Heterocyclic Chemistry, Pergamon Press, Oxford, pp-291-297, 1984.

5. Schilf, W.; Stefaniak, L.; Witanowski, M.; Gebb, G.A., Bull. Pol. Acad. Sci, Chem. 1985, 33, 437.

6. Witanowski, M. ; Stefaniak,L., Bull. Pol. Acad. Sci, Chem. 1987, 35, 305.

7. López, C.; Claramunt, R.M.; Trofinenko, S.; Elguero, J., Can. J. Chem. 1992, 71, 678-684. http://dx.doi.org/10.1139/v93-092

8. Janic, I.; Kakas, M., Vest. Slov. Kem. Drus., 1987, 33(Suppl.), 171-179.

9. Catalán, J.; del Valle, J.C.; Claramunt, R.M.; Boyer, G.; Laynez, J.; Gómez, J.; Jimenez, P.; Tomas, F.; Elguero, J., J. Phys. Chem. 1994, 98, 10606-10612. http://dx.doi.org/10.1021/j100092a035

10. Catalán, J.; de Paz, J.L.G.; Elguero, J., J. Chem. Soc. Perkin Trans. 1996, 2, 57-60.

11. Jalviste, E.; Dziarzhytski, S.; Temps, F., Z. Phys. Chem. 2008, 222, 695-714. http://dx.doi.org/10.1524/zpch.2008.5347

12. Nicken, H.; Temps, F.; Jalviste, E., Z. Phys. Chem. 2011, 225, 1457-1469. http://dx.doi.org/10.1524/zpch.2011.0197

13. Saha, S.K.; Dogra, S.K., J. Photochem. Photobiol.A: Chemistry 1997, 110, 257-266. http://dx.doi.org/10.1016/S1010-6030(97)00192-5

14. Noda, M.; Hirota, N., J. Am. Chem. Soc. 1983, 105, 6790-6794. http://dx.doi.org/10.1021/ja00361a005

15. Nota, M.; Hirota, N.; Sumitani, M.; Yoshihara, K., J. Phys. Chem. 1985, 89, 399-402. http://dx.doi.org/10.1021/j100249a005

16. Mauret, P.; Fayet, J.P.; Fabre, M., Bull. Soc. Chim. Frans, 1975, 7-8; 1675-1678.

17. Catalán, J., J. Phys. Chem. B 2009, 113, 5951-5960. http://dx.doi.org/10.1021/jp8095727 PMid:19344103

18. Catalán, J.; Hopf, H., Eur. J. Org. Chem.2004, 4694-4702. http://dx.doi.org/10.1002/ejoc.200400311

19. Catalán, J.; Diaz, C., Liebigs Ann. 1997, 1941-1949 and Liebigs Ann.1999, 885-891. 
20. Catalán, J.; Díaz, C.; López, V.; Pérez, P.; de Paz, J.L.G.; Rodríguez, L.G., Liebigs Ann. 1996, 1785-1795.

http://dx.doi.org/10.1002/jlac.199619961112

21. Abe, T., Bull. Chem. Soc. Japan 1965, 38, 1314-1320 and ibid.1966, 39, 936-943.

22. Berden, G.; Meerts, W.L.; Jalviste, E., J. Chem. Phys. 1995, 103, 9596-9606.

http://dx.doi.org/10.1063/1.469974

23. de Paz, J.L.G. personal communication.

24. Catalan, J.; de Paz, J.L.G.; Reichardt, C., J. Phys. Chem. A 2010, 114, 6226-6234. http://dx.doi.org/10.1021/jp1009302 PMid:20446695 